\title{
ANECDOTES AND EXPERIENCES
}

\section{Computers in spatial vision research}

\author{
GERALD WESTHEIMER \\ University of California, Berkeley, California
}

\begin{abstract}
The original impulse for using computers in a laboratory dedicated to spatial vision research and the current role of computers in experiments are detailed. Although computers are used widely for modeling spatial vision, there is some doubt about whether conventional serial computers are the most appropriate tools for this task.
\end{abstract}

One look into any vision laboratory reveals that computers have become an integral part of the armamentarium of the researcher. Their impact on research procedures must rival that of the introduction, more than a century ago, of electricity to replace candles as a light source. Computers were available, at high cost and at remote locations, at least 30 years ago, but it is only within the last decade or so that they have been introduced into the laboratory. On-line control of experiments by computers is now standard, and the coupling of computers to lightgenerating devices such as cathode-ray tubes (CRTs) has made possible previously undreamed-of methods of stimulus presentation.

We shall not concern ourselves here with the radical changes that have already taken place in modes of scientific communication between vision researchers, and the much more dramatic changes that are bound to come. There are trivial examples, such as the capability to reformat a manuscript almost instantaneously when change is mandated by the journal to which it is submitted. More significant is the extensive use of computerized data bases and publication procedures that should be fully implemented within a decade or so. The essence of the scientific enterprise is not likely to be seriously affected, of course, because genuinely new ideas and significant concepts will stand out regardless of the volume of trivia within which they appear. Still, signal-to-noise ratio is a factor in scientific communication that cannot entirely be neglected.

\section{THE COMPUTER IN THE VISION LABORATORY}

Many tales can be told of how computers have transformed all aspects of vision experiments. My own experiences in this connection may just be typical, but for me the changes were all but revolutionary. Not that I was

The author's mailing address is: Gerald Westheimer, Department of Physiology-Anatomy, University of California, Berkeley, CA 94720. a stranger to automatizing data-taking. In the early $1960 \mathrm{~s}$ I connected a digital voltmeter and universal timer to a printer. To record intensity discrimination thresholds by the method of adjustment, I attached a neutral density wedge to a good linear potentiometer and arranged the apparatus so that a single buttonpress recorded the time, the control parameters, and the voltage on the printer. In this way, I dispensed with one of the two dramatis personae always present in the older literature of experimental psychology - the experimenter. Only the observer was needed. There was, incidentally, a compelling reason for this approach: even in those early days in Berkeley, I could never be certain whether my student research assistant would show up on any Saturday morning. (The reason for doing research on Saturdays was, of course, that I gave anywhere from 6-10 lectures during the week, in addition to actually supervising laboratories.) Automated datataking and acting as my own observer thus made it possible to get things done.

When fine spatial discrimination, now called hyperacuity, captured my attention about 1966, vision researchers faced formidable technical obstacles in experimentally validating that the human visual system can discriminate spatial positions down to a couple of seconds of arc. A second of arc is a distance of $1 \mathrm{~mm}$ seen at $200 \mathrm{~m}$. When Stratton tested this capability at the turn of the century in Berkeley (Stratton, 1902), he utilized the then uncluttered landscape and clear atmosphere, and had his observers look out of the window of one building to view the stimulus pattern shown in the window of another building $120 \mathrm{~m}$ away. Our experimental arrangement was a little more modern, but not much. We set up the apparatus in the attic of the old optometry building, which allowed us an unobstructed observation distance of $43 \mathrm{~m}$. The experimenter set the pattern position by hand, using a micromanipulator and a table of random numbers. The observer's responses, communicated via an intercom, were recorded by hand. The important thing, of course, was that we could replicate Ludvigh's (1953) claim that vernier acuity for two dots was as good as that for two lines. 
When I became seriously interested in the problem in 1972, the PDP-11 computer (Digital Equipment Corporation) had just been released and I was fortunate in being able to get a rather full version, with the GT- 40 vector display and the 12-bit digital-to-analog scope drivers. The vector display unit has a positioning precision of $1,024 \times 768$ and can draw a line in about $40 \mu \mathrm{sec}$ at one of eight brightness levels. The D/A converters drive a pair of Tektronix 602 display CRTs, which have the virtue of being linear and extremely stable. The main computer has a programmable clock and a 16-bit input/output port, permitting the user to read in switch settings and put out control pulses where needed. There is also an analog-to-digital converter for experiments with the method of adjustments, but it has not been used very much.

When we first acquired the machine, the higher order languages FORTRAN and BASIC could not properly address the display devices, so from the outset we got used to writing our immediate display instructions in assembly language. The absolute control this has given over the temporal and spatial parameters of the stimulus has been an invaluable component of our work, and I have insisted on programming the actual display in assembly language in all systems since. In $1974 \mathrm{I}$ learned how to make these machine language display routines into FORTRAN subroutines and developed a rather elaborate shell program with randomized parameters, automatic disk storage of data, analysis of results by a resident probit program, and other features.

Within the span of a year, then-it took us just about that long to understand the machine's full capabilities and develop the complete program system-the face of our vision laboratory was radically altered. Stimulus presentation, sequencing and randomization, response recording, and result analysis were now entirely automatic, and this allowed the experimenter to be his or her own observer without in any way prejudicing the outcome. Most important, however, was the exquisite control we had over the spatial parameters of the visual stimulus. At an observation distance of $6.8 \mathrm{~m}$ (usually secured by having one first-surface mirror in the room), the GT-40 can be addressed to give individual locations of $6 \mathrm{sec}$ of arc, although the line width is at least double that. The bulk of our research on hyperacuity (Westheimer, 1981) has been obtained with this set-up, with which we can vary specific components of the stimulus, change their relative timings or luminances, give them movements, and so on. Experiments of this kind would have been quite inconceivable had we had to generate the stimulus with more conventional light sources and mechanical control of position and movement. This is even more true for the study of stereoscopic vision. The computer allows us to position displays separately on two CRTs with a precision of 1 in 4,096 in two dimensions. There is no vector capability-each spot is brightened individually-but if we pay careful attention to the display strategy, several hundred dots can be generated in each refresh cycle of $10 \mathrm{msec}$. It should be emphasized that both arrangements, the GT-40 and the digital-to-analog converters coupled to the Tektronix units, are $x / y$ and not raster scan displays. With raster scan displays, the user is locked into a $30-\mathrm{Hz}$ (or, more recently, 60-Hz) refresh rate that makes it virtually impossible to investigate certain temporal discrimination capabilities with which the human visual system can demonstrate sensitivities in the millisecond range.

An essential practical aspect of this research methodology needs to be mentioned. The user can never take for granted that the complex electronic and electro-optical machinery involved in all this actually does what it has been instructed to do. We have made it a mandatory part of our research routine to verify the display on every occasion. We keep on hand a ruler and a photomultiplier with an oscilloscope having a calibrated time base, and we frequently monitor the distances in the stimulus patterns and their durations.

Thus a radical transformation has taken place in the vision research laboratory as a consequence of the introduction of an affordable minicomputer. It is true that the basic research method has not changed: the psychophysical investigation of human spatial discrimination capabilities goes on as before, with carefully designed stimuli and threshold measurements. But the ease of stimulus presentation and data acquisition afforded by a resident dedicated computer has greatly reduced what may be called the turnaround time, that is, the time that elapses between the conception of a particular experiment and the achievement of results. A decade earlier, this period may have been 6 months; the minicomputer reduced it to 6 days or even 6 hours. In our laboratory, we have capitalized on this reduction by leaving unchanged the time spent on research for a publication and increasing the amount of ground covered. It follows that we have been able to pose and answer perhaps an order of magnitude more questions than could be addressed in the same time period before the advent of the computer. I believe that the greater insight into the mechanisms of human spatial discrimination that we have achieved is the direct result of an increased concentration of attention and an increased willingness to engage in trial-and-error forays, without any associated increase in personnel.

Before turning to the next topic, I must add a footnote to the above story. Although the decade between the mid-1960s and the mid-1970s saw enormous advances in the instrumentation available for this kind of psychophysical vision research, a decade later-that is, at the time of this writing -the laboratory is virtually unchanged. Suitable computers have become much cheaper, but no faster. (I am referring here to a single-user installation that can be dedicated to the task. Large mainframe computers are not suitable for this research because the experimenter needs continuous immediate access during critical phases of the stimulus display. The settling time of dig:tal-to-analog converters is on the order of a few microseconds, which is also the time per instruction of the earlier PDP-11 and the current better microcomputers. One need not go any faster, but one cannot go much more 
slowly, either.) A new generation of raster scan displays may change this picture, but these should have at least $1,000 \times 1,000$ pixels, at several intensity levels and colors. The memory capabilities and loading speeds will need to be much higher. The next full generation of microcomputers for psychophysical vision research has not yet appeared.

\section{COMPUTER MODELING OF THE VISUAL SYSTEM}

The use of computers for modeling the visual system is very popular at present. And not undeservedly, because there is a widely perceived need for automatic analysis of visual scenes for pattern recognition and robotics. Just as it became apparent a while ago that language translation is not a task that can simply be relegated to a computer, it is now realized that the recognition of even simple objects is beyond the reach of ordinary machines and programming techniques. This has led to a flowering in the field of artificial intelligence (AI), attracting some of the best minds to it. David Marr was one of these, and just before his early death he left us a book called simply Vision (1982). Its overriding significance is in its emphasis on the search for what one may call the grammar of human vision, as a preparatory step to programming machines to "see." It is not sufficient to try machine translation by merely working with the individual letters or even words of the two languages; similarly, the enumeration of the brightness, color, and position in time of individual spots in a picture is not sufficient for the identification of complex objects, faces, or even single letters of the alphabet from an unknown type set.

Marr (1982) was enthusiastic and optimistic about the possibility of gleaning the grammar of visual perception from psychophysical data. But when we look at the actual results of his studies we are disappointed. The reason lies in Marr's use and interpretation of the papers he read from the literature. They were, by and large, the products of experiments done in an earlier era, when we worked in the attic, or even when Stratton had his observers look from a window across the campus at a stimulus pattern in another building. The results were usually incontestable, but because of the restrictions imposed on the breadth of these studies by the available instrumentation and time, they were limited and should never have been regarded as sufficient substrate for models intended for computer implementation. What appeared to Marr to be a plausible model turns out, on closer examination and more detailed experimental probing, to be inadequate to support more general theories.

An example of these inadequate studies is the formulation of spatial vision in terms of channels. The idea was borrowed from the field of color vision. It is a biophysical fact that light arriving at the retina is spectrally filtered inside the cones. All photopic light signals bear one of only three color signatures. Appropriate subsequent processing permits us to name and distinguish between a wide palette of hues, but there are only three kinds of original neural signals. That our visual system is trichromatic has been known for hundreds of years. Until recently, one of the strongest lines of evidence for trichromacy came from adaptation experiments, in which the temporary sensory deficits that result from strong specific chromatic stimulation are measured. When similar effects were discovered for spatial light patterns in which prolonged viewing of coarser patterns caused an increase in increment thresholds that was confined predominantly to patterns of that size, it was, therefore, in the tradition of visual science to postulate that spatial vision proceeded also via "channels." Accordingly, Marr and others, when they developed models of human vision for computer implementation, assumed that early spatial processing was size specific, that is, that in a given retinal region the signals from a large blobby feature were kept separate from signals from, for example, a small sharp feature. Visual perception thus was thought to develop from space- and size-specific primitives.

The impetus for this kind of rather explicit modeling came from AI needs. Machine vision is an almost intractable subject. Any hint from the description of human vision is exceedingly welcome. Why not work out a procedure for recognizing certain objects by filtering the image through overlying size-specific templates? How many templates and what sizes? Refer to papers on human spatial channels in the journal Vision Research.

But it takes a rather practiced eye to distinguish in these papers, which are based usually on impeccable experimental results, between conclusions that are merely plausible and those that are strongly compelling. It is a hazard that those of us who have long familiarity with the field are well aware of, but that can easily trap the physicist, engineer, or mathematician new to the field of spatial vision research. Claims for channel processing in spatial vision, when read with more discrimination, soon lose their appeal as universally valid postulates.

The computer is a central issue here. In earlier days, when the grim task of the actual calculation needed to create a mathematical model acted as a deterrent to all but the facile (and they had better things to do), we were usually given only mere hints of how a final model might work. Now the computations are easily done by simple numerical methods, rather than the more demanding analytical ones. Additional components can be added at will to secure a good fit. The result frequently is an appealing model that we are tempted to regard as a fair blueprint of how the human sees, and therefore of how we ought to build a robot capable of recognizing objects. But did the original data have enough substance to carry the load of the model?

The core of the problem most often is a view of the general validity of a set of psychophysical results that is too optimistic, no matter how expertly these results were obtained. The mammalian nervous system has a degree of complexity, of overlying and intertwining connections, that defies quick general statements in all but the most 
basic instances. Examples of these basic instances are the trichromacy of color vision and the funneling of spatial vision through individual receptors. (Even here caveats and doubts enter: The human photopic pigments are grouped in families rather than seen as three specific ones; anatomical bridges between receptors may make them act partially in concert, rather than purely individually.) The range of applicability of a particular psychophysical curve is sure to be limited, a fact that the very nature of the research enterprise tends to downplay rather than highlight. Strangely, the computer, whose facility with numerical data and functions prompted us to use as firm building blocks data that are really only tentative psychophysical indicators, can also help to rescue us from the dilemma it created. Because it allows considerably faster acquisition of psychophysical data, it encourages the acquisition of more data. Invariably, the complexity of the situation then surfaces and a more lenient, less succinct, less numerically precise picture of the human visual system emerges-in short, a view that does justice to the deep complexity of the human nervous system instead of merely caricaturing it.

In closing, it might be observed that all this was foreseen in the dawning of the age of computers by its single most influential genius, John von Neumann. On his deathbed, von Neumann (1958) sketched the manuscript of his Silliman Lectures entitled The Computer and the Brain. Even as the first modern computers were being built, von Neumann understood the fundamental difference between them and the brain. His writing on this point is not quite as precise as we would wish-the manuscript was finished by his wife after he died. But we can now understand very well what he intended to say. The operation of the nervous system proceeds along lines characterized by much less arithmetical depth than that provided in computers. I think I know what von Neumann meant. I once tried to calculate Bessel functions by means of the expansion formulas, using a version of BASIC with only six decimal places. This method soon gave complete nonsense answers, or rather, it gave neatly ordered answers that bore no relationship to the Bessel functions in the published tables.The neurons of the nervous system provide at best two orders of precision. Because the information flow proceeds essentially in a cascaded fashion, it follows that the brain, whatever it does, will not exhibit the arithmetic precision expected of a computer. Phrasing the description of the human visual system by formulations that imply high arithmetic precision is, therefore, a mismatch. Insofar as models are geared to the essential capabilities of modern serial computers, with their great arithmetical and logical depth, that is, retention of precision through many logical steps, the mismatch is compounded.

Before spending any great effort on theoretical frameworks for the human visual system, the researcher needs to confront von Neumann's ultimate thoughts on computers and the brain. Sensible benchmarks always exist, of course. In machine vision it is not the elegance of the formulation that is important, but how well the device works. Human vision presents a much bigger challenge. The success story of contemporary computers should not deflect us from meeting this challenge on its own terms. It demands an approach that is at once more supple, more varied, more textured, more diversely interactive, more subject to instantaneous and long-term modification, than anything that can be compressed into programs for the current generation of computers.

\section{REFERENCES}

LuDVIGH, E. (1953). Direction sense of the eye. American Journal of Ophthalmology, 36, 139-142.

MARR, D. (1982). Vision. San Francisco: W. H. Freeman.

Stratton, G. M. (1902). Visible motion and the space threshold. Psychological Review, 9, 433.

von Neumann, J. (1958). The computer and the brain. New Haven: Yale University Press.

Westheimer, G. (1981). Visual hyperacuity. Progress in Sensory Physiology, 1, 1-30. 